\title{
Tuning Transport Properties of Topological Edge States of Bi(111) Bilayer Film by Edge Adsorption
}

\author{
Z. F. Wang, ${ }^{1}$ Li Chen, ${ }^{2,3, *}$ and Feng $\operatorname{Liu}^{1,4, \oplus}$ \\ ${ }^{1}$ Department of Materials Science and Engineering, \\ University of Utah, Salt Lake City, UT 84112 \\ ${ }^{2}$ Institute of Condensed Matter Physics, Linyi University, Linyi, Shandong 276005, China \\ ${ }^{3}$ State Key Laboratory of Low Dimensional Quantum Physics and \\ Department of Physics, Tsinghua University, Beijing 100084, China \\ ${ }^{4}$ Collaborative Innovation Center of Quantum Matter, Beijing, China
}

\begin{abstract}
Based on first-principles and tight-binding calculations, we report that the transport properties of topological edge states of zigzag $\mathrm{Bi}(111)$ nanoribbon can be significantly tuned by $\mathrm{H}$ edge adsorption. The Fermi velocity is increased by one order of magnitude, as the Dirac point is moved from Brillouin zone boundary to Brillouin zone center and the real-space distribution of Dirac states are made twice more delocalized. These intriguing changes are explained by an orbital filtering effect of edge $\mathrm{H}$ atoms, which removes certain components of $p$ orbits of edge $\mathrm{Bi}$ atoms that reshapes the topological edge states. In addition, the spin texture of the Dirac states is also modified, which is described by introducing an effective Hamiltonian. Our findings not only are of fundamental interest but also have practical implications in potential applications of topological insulators.
\end{abstract}

PACS numbers: 73.43.-f, 73.22.-f, 85.75.-d, 75.70.Tj

Studies of chemical and structural edge modification of 2D materials are of great scientific and technological interest because such edge modification are expected to significantly change the properties of $2 \mathrm{D}$ structures, especially 2D nanostructures because of a large edgeto-surface ratio, in analogy to surface modification of 3D nanostructures with a large surface-to-volume ratio. One well studied example is graphene nanoribbons (GNRs) [1 4]. However, much less attention has been paid to edge modification of $2 \mathrm{D}$ topological insulators (TIs). This is because the topological nature of TI edge states, with a origin from bulk band topology, is wellknown to be robust, insensitive to nonmagnetic chemical and structural edge modification [5, 6]. Especially, some fundamental topology-defined transport properties of TI edge state, such as its quantized edge conductance and spin-momentum locking relation, cannot be changed. On the other hand, other characteristic transport properties of TI edge state, such as carrier mobility, the number of quantum conductance and the spin texture, can all in principle be changed by edge modification, but modification of transport properties of TI edge state has been rarely demonstrated.

Ultrathin $\mathrm{Bi}(111)$ films are theoretically predicted to be a 2D TI material [7 11], which have been experimentally synthesized and characterized recently 12 15]. In this Letter, we demonstrate that the transport properties of TI edge states in zigzag $\mathrm{Bi}(111)$ nanoribbon (ZBNR) can be tuned by chemical edge modification via $\mathrm{H}$ adsorption. Most remarkably, the Fermi velocity of Dirac edge state is increased by as much as one order of magnitude, when the Dirac point is moved from the Brillouin zone boundary to Brillouin zone center. Correspondingly, the real-space distribution of Dirac states is found to be much more delocalized. In addition, the spin texture of the Dirac states is also modified, with the spin orientation switching from predominantly in-plane to out-of-plane alignment around the Dirac point. Through a systematic analysis based on model first-principles and tight-binding (TB) calculations, the physical mechanism underlying these intriguing phenomena is revealed to be the atomic orbital filtering effect by edge $\mathrm{H}$ atoms. The edge $\mathrm{H}$ removes part of $\mathrm{x}$ - and $\mathrm{z}$-components of $p$ orbits of the edge $\mathrm{Bi}$ atoms, leading to a change of edge boundary potential that reshapes the topological edge states. The change of spin texture is further analyzed by an effective Hamiltonian.

The first-principles calculations of ZBNR, containing eighty $\mathrm{Bi}$ atoms, including spin-orbit coupling and without/with $\mathrm{H}$ adsorption are carried out in the framework of generalized gradient approximation with Perdew-Burke-Ernzerhof functional using the VASP package [16]. The supercells have a vacuum layer more than $15 \AA$ thick to ensure decoupling between neighbouring ZBNRs. All self-consistent calculations are performed with a plane-wave cutoff of $400 \mathrm{eV}$ on an $1 \times 11 \times 1$ Monkhorst-pack k-point mesh. For structural relaxation, all the atoms are allowed to relax until atomic forces are smaller than $0.01 \mathrm{eV} / \AA$. The TB calculations of ZBNR are done using the Wannier90 package [17]. First, the TB Hamiltonian of one unit cell, containing two $\mathrm{Bi}$ atoms and twelve maximally localized Wannier functions (MLWFs) of $p$ orbits, is fitted to the first-principles calculations. Using this unit-cell TB Hamiltonian, we further constructed a supercell TB Hamiltonian of ZBNR, containing eighty Bi atoms. To model $\mathrm{H}$ adsorption effect, $p$ orbits of edge $\mathrm{Bi}$ atoms are selectively removed in the TB calculations.

Figures 1(a) and 1(b) show the first-principles band structures of ZBNR without and with edge $\mathrm{H}$ adsorption, respectively, illustrating the remarkably different topological edge states under different chemical edge environments. Without $\mathrm{H}$ adsorption [Fig. 1(a)], there are two extended gapless edge states inside the bulk 
band gap. The two edge states, connecting the valence and conduction bulk bands (shaded yellow regions) and forming a 1D Dirac state at the Brillouin zone boundary, are characterized by an odd number of crossings over the Fermi level (from $k_{y}=0$ to $k_{y}=1$ ). Tuning the Fermi level within the band gap, the number of crossings can be either three or one, indicating the topological nature of ZBNR. Due to the inversion symmetry, each edge state is degenerated for the left and right edges. The real-space distribution of these degenerated edge states at four chosen k-points [as marked in Fig. 1(a)] are shown in Fig. 1(c). We see that the width of localized edge state is k-point dependent and its maximum width is $\sim 2 \mathrm{~nm}$, which is consistent with previous scanning tunneling microscopy (STM) measurement [13].

However, with $\mathrm{H}$ adsorption at the edge of ZBNR, the topological edge states are dramatically modified in three important ways, as shown in Figs. 1(b) and 1(d). First, the most significant finding is that the Fermi velocity $\left(\nu_{F}\right)$ of Dirac states, as obviously reflected by the band slope, is increased by as much as one order of magnitude, changing from $1.1 \times 10^{5} \mathrm{~m} / \mathrm{s}$ to $0.9 \times 10^{6} \mathrm{~m} / \mathrm{s}$. This high Fermi velocity is comparable to the largest $\nu_{F}\left(3 \times 10^{6} \mathrm{~m} / \mathrm{s}\right)$ obtained from suspended graphene [18]. The Fermi velocity is one of the key parameters in the study of Dirac materials, as it bears various fundamental information, such as electron mobility, electron-electron interaction [18] and effective fine structure constant [19]. Several different routes have been proposed to engineer $\nu_{F}$ in graphene [20], but few have been reported in TIs. Here, we demonstrate that chemical edge modification presents a simple and powerful way to engineer $\nu_{F}$ in 2D TIs.

Second, the original extended gapless edge states become localized at Brillouin zone center in the $k$-space, similar to the edge states in $\mathrm{HgTe} / \mathrm{CdTe}$ quantum well [8]. Consequently, there is only one crossing point between the edge state and Fermi level within the energy window of bulk band gap. This modification can have useful implications in spintronic devices. With one crossing, electron backscattering is in principle completely forbidden. Having three crossing points the edge state and Fermi level, electron backscattering can no longer be $100 \%$ prohibited in a scattering process between two states with same energy but different momenta. This is because the spin directions of those two states are not completely inverse with each other [13].

Third, the real-space distribution of the localized edge states become much wider than those without $\mathrm{H}$ adsorption, as seen by comparing Fig. 1(d) with Fig. 1(c). As k-point moves away from the Dirac point, the localized edge states become more and more delocalized, whose width of distribution increases to a maximum of $\sim 4.4 \mathrm{~nm}$. This observation is consistent with previous theory of penetration depth of edge states, which is inverse to k-space distribution of the edge states 8]. A localized state in momentum space will have an extended distribution in real space.

The results shown in Fig. 1 demonstrate the feasibil-
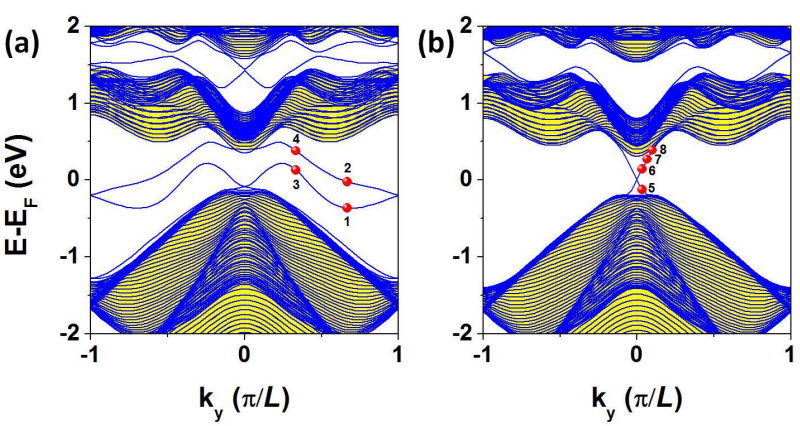

(c) 1

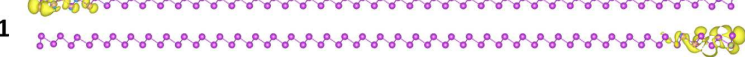

अ

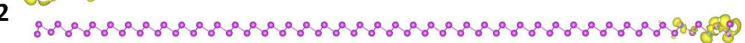

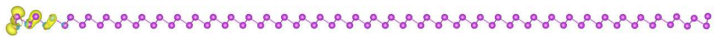

3

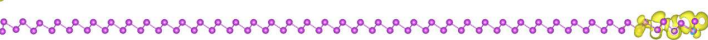

4

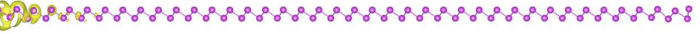

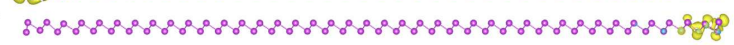

(d)

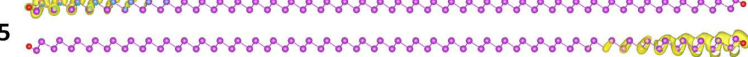

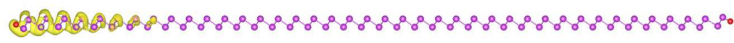

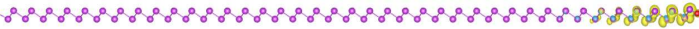
आ영여영 7 .

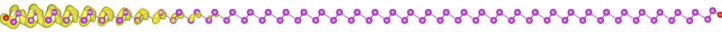

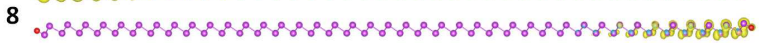

FIG. 1: (a) and (b) First-principles band structures of ZBNR without and with edge $\mathrm{H}$ adsorption, respectively. The shaded yellow regions are bulk states with a band gap; the solid blue lines inside the band gap are topological edge states. (c) and (d) Side view of real-space charge density distributions of the edge states at different k-points, as marked in (a) and (b), respectively.

ity of tuning some important characteristic transport properties of 2D TI edge states by chemical edge modification. Next, we perform an orbital analysis to reveal the physical mechanism underlying this phenomenon. There are one $s$ orbit for each $\mathrm{H}$ atom and three $p$ orbits for each Bi atom, as schematically shown in Fig. 2 (a). At the ribbon edge, each Bi atom is bonded with a $\mathrm{H}$ atom. Structural optimization confirms that the $\mathrm{H}$-Bi bond is within the $\mathrm{z}-\mathrm{x}$ plane; i.e. it is perpendicular to the $p_{y}$, but not perpendicular to the $p_{x}$ or $p_{z}$ orbit of Bi atom. Considering the orbital symmetry, it is easy to see that the hopping between $s$ orbit of $\mathrm{H}$ and $p_{y}$ orbit of $\mathrm{Bi}$ is zero, but the hopping between $\mathrm{s}$ orbit of $\mathrm{H}$ and $p_{x}$ (or $p_{z}$ ) orbit of $\mathrm{Bi}$ is not zero. Therefore, the effect of $\mathrm{H}$ atoms is to partially saturate $p_{x}$ and $p_{z}$ orbits of edge $\mathrm{Bi}$ atoms.

In order to support such hypothesis, we have further performed several first-principles and TB model calculations. First, we construct a TB Hamiltonian of ZBNR using the ideally bulk-terminated edge without edge structural relaxation [Fig. 2(b)]. The band structures obtained from the first-principles and TB model calculations are shown in Figs. 2(c) and 2(d), respectively. We see good agreement between the two 
(a)

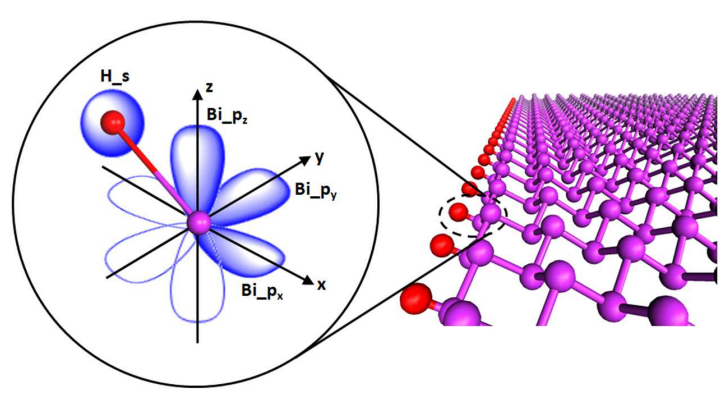

(e)

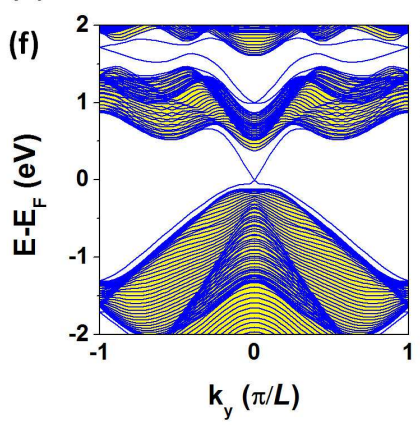

(b)
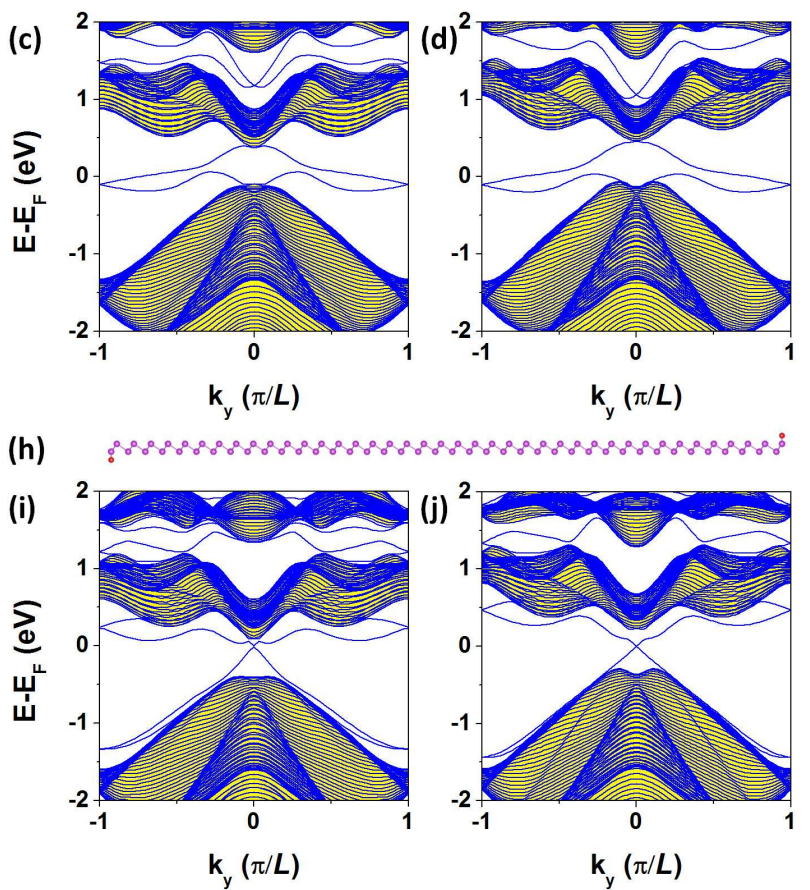

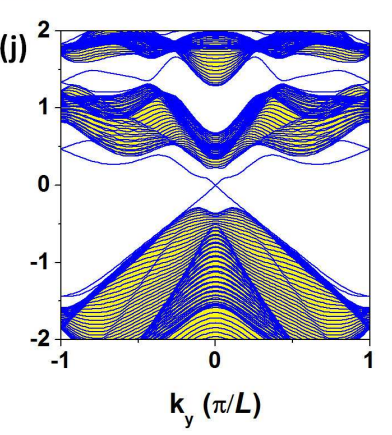

FIG. 2: (a) Illustration of the orbit and bonding at the edge of ZBNR with $\mathrm{H}$ adsorption. One s orbit for each $\mathrm{H}$ atom and three $p$ orbits for each Bi atom are shown. (b), (e) and (h) are the atomic structure of ZBNR without H adsorption, with $\mathrm{H}$ adsorption along $\mathrm{x}$-direction and with $\mathrm{H}$ adsorption along $\mathrm{z}$-direction, respectively. The atomic structures are not relaxed. (c), (f) and (i) are the first-principles band structures of (b), (e) and (h), respectively. (d), (g) and (j) are the TB band structures of (b), (e) and (h), respectively.

methods for both bulk and edge states, validating the effective TB Hamiltonian. Next, we artificially fix the adsorbed $\mathrm{H}$ atoms along either $\mathrm{x}$ - or z-direction without structural relaxation, while maintaining the inversion symmetry of the ribbon, as shown in Figs. 2(e) and $2(\mathrm{~h})$, respectively. This special setup allows us to selectively saturate either $p_{x}$ or $p_{z}$ orbit of the edge Bi atoms. Thus, we can distinguish the effect of those two orbits from each other. The $\mathrm{H}-\mathrm{Bi}$ bond length is set to $1.82 \AA$, as obtained from the relaxed structure in Fig. 1(d).

The first-principles band structures for the above two different $\mathrm{H}$-adsorbed model configurations are shown in Figs. 2(f) and 2(i), respectively. The most significant finding is that a new Dirac state appears at Brillouin zone center in both cases. In the x-direction $\mathrm{H}$-adsorbed configuration when the $p_{x}$ orbit of edge $\mathrm{Bi}$ atoms is removed [Fig. 2(f)], the upper branch of the new Dirac state spans the whole energy window of the bulk band gap, while the lower branch of the new Dirac state almost merges into the bulk valence bands. However, in the z-direction $\mathrm{H}$-adsorbed configuration when the $p_{z}$ orbit of edge $\mathrm{Bi}$ atoms is removed [Fig. $2(\mathrm{i})$, the lower branch of the new Dirac state spans the most energy window of bulk band gap. Both upper and lower branches of the new Dirac state have an extended dispersion, resulting in two additional Dirac states at Brillouin zone boundary.

To more clearly see the $\mathrm{H}$ adsorption effect in above two configurations, we also selectively removed the $p_{x}$ or $p_{z}$ orbit of edge $\mathrm{Bi}$ atoms in the TB Hamiltonian. This can be done by adding a large on-site energy on these selected orbits, similar to a method used previously to study the gapless edge state in GNR 21]. The corresponding TB band structures are shown in Figs. $2(\mathrm{~g})$ and $2(\mathrm{j})$ for removing $p_{x}$ and $p_{z}$ orbit, respectively, which are consistent with the first-principles results of Figs. 2(f) and 2(i). The comparison between the first-principles and TB calculations indicates that the $\mathrm{H}$ atoms act as an orbital filter [11], which selectively removes the orbit of edge $\mathrm{Bi}$ atoms and reshape the topological edge states. It is the combined effect of partial removal of both $p_{x}$ and $p_{z}$ orbits of edge $\mathrm{Bi}$ atoms that is responsible for generating the new gapless Dirac edge states in Fig. 1(b) by $\mathrm{H}$ edge adsorption.

It is interesting to note that if both $p_{x}$ and $p_{z}$ orbits of edge $\mathrm{Bi}$ atoms were completely removed simultaneously, e.g., by adsorbing two $\mathrm{H}$ atoms on the edge $\mathrm{Bi}$ atoms, the Dirac state would remain located at Brillouin zone boundary, as shown in Fig. S1(a) [22]. Also, the effect of removing $p_{y}$ orbit of edge $\mathrm{Bi}$ atoms has been studied. It would reshape the edge states and induce two Dirac states at Brillouin zone boundary, as shown in Fig. S1(b) [22]. However, practically it is hard to find a way to just remove the $p_{y}$ orbit. Furthermore, if there is no chemical adsorption, structural relaxation alone will not change the position of Dirac point, and hence without significantly changing the shape of edge state. This can be seen by comparing 
the first-principles band structures of ZBNR with [Fig. 1(a)] and without [Fig. 2(c)] structural relaxation.

Next, we explain where the new gapless Dirac edge state at Brillouin zone center comes from. The TB model provides us with an easy method to analyze the evolution of topological edge states upon edge modification. We can continuously remove $p_{x}$ and $p_{z}$ orbits of edge $\mathrm{Bi}$ atoms by increasing their on-site energies gradually in the TB Hamiltonian. As shown in Figs. 3(a)-(c), by gradually increasing the on-site energy of $p_{x}$ orbit, the edge states are continuously reshaped. The upper branch of the old Dirac state merges into the conduction bands, and the lower branch of the old Dirac state becomes the upper branch of the new Dirac state, locating the Dirac point at Brillouin zone center. However, the case for $p_{z}$ orbit is a little different, as shown in Figs. 3(d)-(f). By gradually increasing the on-site energy of $p_{z}$ orbit, the edge states are continuously moved upward. Part of the old Dirac state merges into the conduction bands, and the new Dirac state is pulled up from the valence bands. The bulk states are not changed in both cases. From this model calculation, we found that depending on the edge potential, the topological edge states can be either regenerated from or merged into the bulk states, always being connected to bulk states.

Lastly, we show that the spin textures of topological edge states can also be modified by chemical edge modification. The zoom-in first-principles band structures and spin textures around the Dirac states without and with $\mathrm{H}$ adsorption are shown in Figs. 4(a) and 4(b), respectively. We see that the spins are within the $\mathrm{z}-\mathrm{x}$ plane and perpendicular to the momentum direction (y-direction), showing the helical nature and spin-momentum locking property. Without $\mathrm{H}$ adsorption [Fig. 4(a)], the in-plane (x-direction) spin component is dominant near the Dirac point. When k-point goes away from the Dirac point, the out-of-plane (zdirection) spin component becomes larger. With $\mathrm{H}$ adsorption [Fig. 4(b)], the out-plane (z-direction) spin component is dominant near the Dirac point. When $\mathrm{k}$ point goes away from the Dirac point, a small in-plane (x-direction) spin component starts to appear. Thus, the spin direction is wrapping along the Dirac state with different momenta in the two cases. Such a spin texture of the 1D Dirac states can be described by the following low-energy effective Hamiltonian,

$$
H=s \hbar \nu_{F} k_{y}\left[\lambda\left(k_{y}\right) \sigma_{x}+\sqrt{1-\lambda^{2}\left(k_{y}\right)} \sigma_{z}\right] .
$$

where $s= \pm 1$ denotes right/left edge, $\nu_{F}$ is the Fermi velocity and $\sigma_{x / z}$ is the pauli matrix. $\lambda \in[-1,1]$; it is a wrapping function of momentum $k_{y}$. If $\lambda=0$, the spin only has z-component. If $\lambda= \pm 1$, the spin only has $\mathrm{x}$-component. In other cases, the spin can have both $\mathrm{x}$ - and $\mathrm{z}$-components, and spin direction is tuned by the value of $\lambda$.

In conclusion, although the topology of TI edge states is robust against structural and chemical edge modification, we show that some important character-
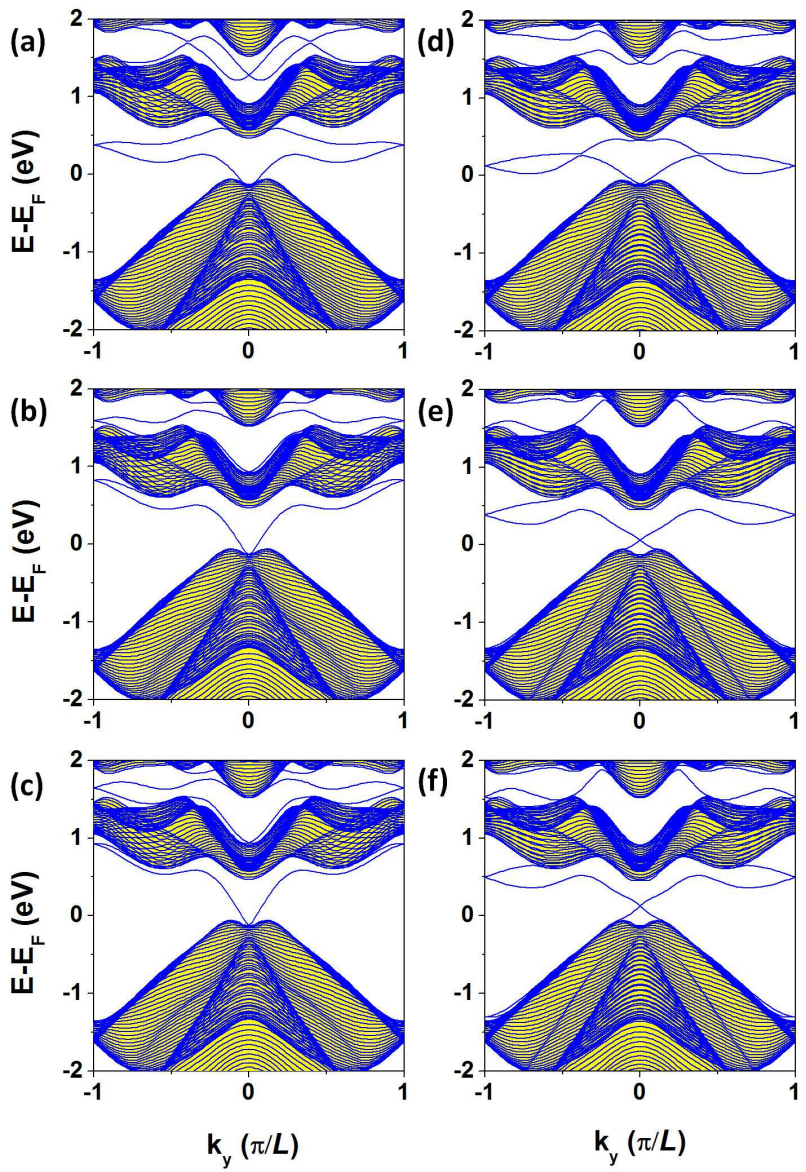

FIG. 3: (a)-(c) Evolution of the topological edge states by gradually removing $p_{x}$ orbit of the edge $\mathrm{Bi}$ atoms. (d)(f) Evolution of the topological edge states by gradually removing $p_{z}$ orbit of the edge $\mathrm{Bi}$ atoms. From (a)-(c) and (d)-(f), the on-site energy for $p_{x}$ and $p_{z}$ orbits are set at 1 , 3 and $5 \mathrm{eV}$, respectively.
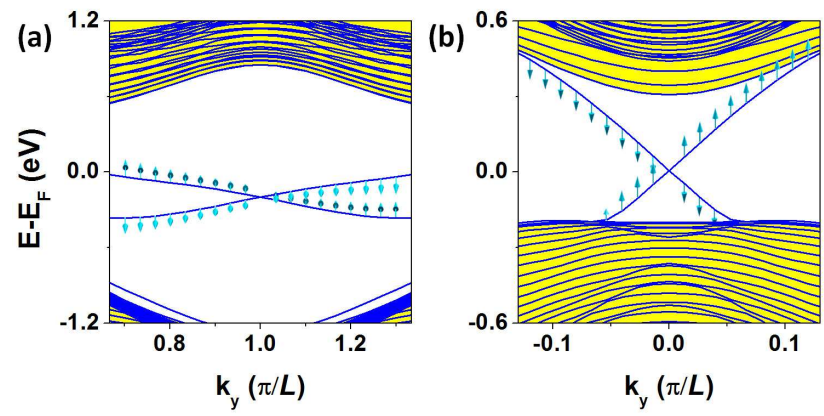

FIG. 4: (a) and (b) Zoom-in band structures around the Dirac point for Fig. 1(a) and Fig. 1(b), respectively. The arrows, perpendicular to momentum, denote the spin textures of edge states. Only the left edge-state spin textures are plotted for each band, which are inverse to the right edge-state spin textures.

istic transport properties of TI edge states can still be significantly modified, as illustrated by $\mathrm{H}$ edge adsorption in ZBNR. Most remarkably, the Fermi velocity can be increased by as much as one order of magnitude, becoming comparable to the largest value found in graphene. Also, the spin textures of TI edge states are modified. Our findings are scientifically interesting for 
a better understanding of basic transport properties of topological edge states in relation to edge boundary conditions, in addition to bulk topology. They also have significant practical implications, because those modified transport properties, such as Fermi velocity and spin texture, are important parameters in TIbased spintronic devices. The approach of engineering topological edge states by chemical edge modification is general, not only applicable to other $2 \mathrm{D}$ TIs but can also be extended to $3 \mathrm{D}$ TIs via chemical surface modification.

* E-mail: lchen.lyu@gmail.com

† E-mail: fliu@eng.utah.edu

[1] Q. Yan, B. Huang, J. Yu, F. Zheng, J. Zang, J. Wu, B.-L. Gu, F. Liu, and W.-H Duan, Nano Lett. 7, 1469 (2007).

[2] B. Huang, F. Liu, J. Wu, B.-L. Gu, and W.-H. Duan, Phys. Rev. B 77, 153411 (2008).

[3] Z. F. Wang, Q. Li, H. Zheng, H. Ren, H. Su, Q. W. Shi, and J. Chen, Phys. Rev. B 75, 113406 (2007).

[4] Z. F. Wang, S. Jin, and F. Liu, Phys. Rev. Lett. 111, 096803 (2013).

[5] M. Z. Hasan, C. L. Kane, Rev. Mod. Phys. 82, 3045 (2010).

[6] X.-L. Qi, S.-C. Zhang, Rev. Mod. Phys. 83, 1057 (2011).

[7] S. Murakami, Phys. Rev. Lett. 97, 236805 (2006).

[8] M. Wada, S. Murakami, F. Freimuth, and G. Bihlmayer, Phys. Rev. B 83, 121310 (2011).

[9] Z. Liu, C.-X. Liu, Y.-S. Wu, W.-H. Duan, F. Liu, and J. Wu, Phys. Rev. Lett. 107, 136805 (2011).

[10] L. Chen, Z. F. Wang, and F. Liu, Phys. Rev. B 87,
$235420(2011)$

[11] M. Zhou, W. Ming, Z. Liu, Z. F. Wang, Y. Yao, F. Liu, arXiv:1401.3392

[12] T. Hirahara, G. Bihlmayer, Y. Sakamoto, M. Yamada, H. Miyazaki, S.i. Kimura, S. Blügel, and S. Hasegawa, Phys. Rev. Lett. 107, 166801 (2011).

[13] F. Yang, L. Miao, Z. F. Wang, M.-Y. Yao, F. Zhu, Y. R. Song, M.-X. Wang, J.-P. Xu, A. V. Fedorov, Z. Sun, G. B. Zhang, C. Liu, F. Liu, D. Qian, C. L. Gao, and J.-F. Jia, Phys. Rev. Lett. 109, 16801 (2012).

[14] L. Miao, Z. F. Wang, W. Ming, M.-Y. Yao, M.-X. Wang, F. Yang, Y. R. Song, F. Zhu, A. V. Fedorov, Z. Sun, C. L. Gao, C. Liu, Q.-K. Xue, C.-X. Liu, F. Liu, D. Qian, and J.-F. Jia, Proc. Natl. Acad. Sci. U.S.A. 110, 2758 (2013).

[15] Z. F. Wang, M.-Y. Yao, W. Ming, L. Miao, F. Zhu, C. Liu, C. L. Gao, D. Qian, J.-F. Jia, and F. Liu, Nature Commun. 4, 1384 (2013).

[16] G. Kresse, J. Hafner, Phys. Rev. B 47, 558 (1993).

[17] A. A. Mostofi, J. R. Yates, Y.-S. Lee, I. Souza, D. Vanderbilt, and N. Marzari, Comput. Phys. Commun. 178, 685 (2008).

[18] D. C. Elias, R. V. Gorbachev, A. S. Mayorov, S. V. Morozov, A. A. Zhukov, P. Blake, L. A. Ponomarenko, I. V. Grigorieva, K. S. Novoselov, F. Guinea, and A. K. Geim, Nature Phys. 7, 701 (2011).

[19] C. Jang, S. Adam, J.-H. Chen, E. D. Williams, S. Das Sarma, and M. S. Fuhrer, Phys. Rev. Lett. 101, 146805 (2008).

[20] C. Hwang, D. A. Siege, S.-K. Mo, W. Regan, A. Ismach, Y. Zhang, A. Zett, and A. Lanzara, Sci. Rep. 2, 590 (2012).

[21] W. Yao, S. A. Yang, and Q. Niu, Phys. Rev. Lett. 102, 096801 (2009).

[22] See Supplemental Material at http://link.aps.org/ supplemental/***/PhysRevLett*** for more information on the orbit effect. 